\title{
Interacting electrons in polygonal quantum dots
}

\author{
C. E. Creffield \\ Department of Physics, King's College London, Strand, London WC2R 2LS, United Kingdom \\ Wolfgang Häusler \\ Universität Freiburg, Fakultät für Physik, Hermann-Herder-Strasse 3, D-79104 Freiburg, Germany \\ J. H. Jefferson \\ DERA, Electronics Sector, St. Andrews Road, Malvern, Worcester WR14 3PS, United Kingdom \\ Sarben Sarkar \\ Department of Physics, King's College London, Strand, London WC2R 2LS, United Kingdom
}

(Received 7 October 1998)

\begin{abstract}
The low-lying eigenstates of a system of two electrons confined within a two-dimensional quantum dot with a hard polygonal boundary are obtained by means of exact diagonalization. The transition from a weakly correlated charge distribution for small dots to a strongly correlated "Wigner molecule" for large dots is studied, and the behavior at the crossover is determined. In sufficiently large dots, a recently proposed mapping to an effective charge-spin model is investigated, and is found to produce the correct ordering of the energy levels and to give a good first approximation to the size of the level spacings. We conclude that this approach is a valuable method to obtain the low-energy spectrum of few-electron quantum dots. [S0163-1829(99)03715-7]
\end{abstract}

The success of modern nanoscale technology in the creation and manipulation of quantum dots ${ }^{1}$ has stimulated considerable theoretical interest in elucidating the physical processes in these structures. In the first analyses exact numerical diagonalizations were carried out to study how many-body effects modify the energy-level spectra, ${ }^{2-5}$ which may be measured by means of nonlinear transport spectroscopy. ${ }^{6}$ As the number of electrons in a quantum dot can be changed in a controlled way down to values of $N$ $=1,2,3 \ldots,{ }^{1,7}$ they can be thought of as "artificial"' $\mathrm{H}, \mathrm{He}$, $\mathrm{Li}$, etc. atoms, composed of interacting electrons confined by an external potential. A vital difference between these structures and real atoms, however, is the importance of correlations. Real atoms can be described to good accuracy by independent electron models within suitable mean-field theories, which can be systematically improved via perturbation theory. This is not generally true for quantum dots for which an independent electron approximation can give results which are not even qualitatively correct. This behavior is a consequence of the relatively small kinetic energies in few electron quantum dots compared with their mutual Coulomb interaction. For sufficiently low-electron densities the Wigner limit will be approached, ${ }^{8,9}$ in which the ground state will adopt a quasicrystalline form to minimize the interaction energy. In nontranslationally invariant systems this can occur at larger "critical" densities than in the homogeneous twodimensional (2D) liquid. ${ }^{10}$

This feature of quantum dots containing low-density electrons has been exploited recently ${ }^{11}$ as a starting point complementary to perturbative or mean-field approaches that are reliable at weak-interaction strengths. As the electron density in these systems localizes around the Wigner lattice points, ${ }^{12}$ it is sensible to construct a many-particle basis from antisymmetrized combinations of nonorthogonal one-particle states localized at these sites. Orthogonalization leads to a lattice model of strongly correlated electrons of Hubbard type, and the subsequent elimination of the high-energy states of this model was then shown to yield an effective " $t J V$ " Hamiltonian, ${ }^{11}$ relevant to the study of electron correlations in real lattice systems, such as the copper-oxide planes of high-temperature superconductors. ${ }^{13}$ This effective Hamiltonian has a very much smaller Hilbert space than that of the original quantum dot enabling, in principle, the treatment of systems of many more electrons than can be handled by direct diagonalization methods. The ordering of the lowenergy levels as well as their relative separations agree quite satisfactorily with data obtained by exact diagonalization for a one-dimensional dot containing up to four electrons. ${ }^{11}$ Here we examine the validity of the $t J V$ approach in twodimensional hard-wall boxes. In the case of two (or higher) dimensions, it should be noted that the number of energy minima in configuration space, i.e., the number of peaks $N_{0}$ in the charge-density distribution in the low-density limit, can be larger than the number of electrons. For example, for two electrons in a square box the ground state will consist of a superposition of states in which the electrons are located on diagonally opposite vertices of the square, and so will exhibit a four-peak structure. In this paper we consider three different box shapes: triangular, square, and hexagonal. For the case of two electrons the eigenenergies and eigenfunctions are obtained by exact diagonalization, and then compared with the predictions of the $t J V$ model.

We consider the Hamiltonian:

$$
H=\sum_{i=1}^{2}\left[-\frac{\hbar^{2}}{2 m^{*}} \nabla_{i}^{2}+V\left(\mathbf{r}_{i}\right)\right]+\frac{e^{2}}{4 \pi \epsilon_{0} \epsilon_{r}} \frac{1}{\left|\mathbf{r}_{1}-\mathbf{r}_{2}\right|},
$$


where we assume that the electrons can be described within the parabolic band approximation by an effective mass $m^{*}$. The shape of the dot is set by the confining potential $V(\mathbf{r})$, which is zero within the dot and infinite outside. The full wave function of the two-electron system may be written as a product of a spinor and a spatial function:

$$
\Psi\left(\mathbf{r}_{1}, \sigma_{1} ; \mathbf{r}_{2}, \sigma_{2}\right)=\psi\left(\mathbf{r}_{1} ; \mathbf{r}_{2}\right) \chi\left(\sigma_{1} ; \sigma_{2}\right),
$$

where for a singlet (triplet) state $\psi$ is symmetric (antisymmetric) under particle exchange. As the Hamiltonian (1) does not contain any spin dependent terms it is only necessary to consider its action on the spatial component of the wave function to obtain its eigenvalues. We choose to use a basis of position eigenstates (a finite-difference method) as opposed to the momentum eigenbasis used, for example, in Refs. 2 and 14. An advantage of using this basis is that it is a simple matter to impose the required homogeneous Dirichlet boundary conditions for dots regardless of their shape, which can be very difficult to achieve in a momentum-space basis. An exception to this is the case of the square shape, which we used to check the reliability of the finite-difference approach by comparing with results obtained using a momentum-space basis. The agreement was found to be excellent.

Using the basis of position eigenstates amounts to replacing the spatial continuum $\left(\mathbf{r}_{1} ; \mathbf{r}_{2}\right)$ with a four-dimensional mesh. The wave-function $\psi$ is then only evaluated at a discrete set of points:

$$
\psi\left(x_{1}, y_{1} ; x_{2}, y_{2}\right) \rightarrow \psi_{i j k l},
$$

and the spatial derivatives in Eq. (1) are replaced with symmetric difference approximations:

$$
\frac{\partial^{2} \psi}{\partial x_{1}^{2}} \rightarrow \frac{\psi_{i+1 j k l}-2 \psi_{i j k l}+\psi_{i-1 j k l}}{(\Delta x)^{2}} \text {, etc. }
$$

The Hamiltonian can now be represented as a $N^{4} \times N^{4}$ matrix, where $N$ is the number of mesh points per dimension. Since the Coulomb interaction is diagonal in this representation, and the kinetic terms (4) only operate between neighboring mesh points, this matrix will clearly be extremely sparse. This allows it to be stored in a very economical fashion, and also permits a highly efficient use of Lanczos diagonalization routines to obtain the lowest few eigenstates. As $N$ is increased, and the separation between the mesh points decreases, the spectrum of the discrete system will approach that of the continuum model and the form of the mesh will be irrelevant. Finite-size effects can be minimized, however, by choosing a mesh that matches the symmetries of the potential as far as possible. For the case of a square dot we therefore chose a square mesh, and a triangular mesh for the triangular and hexagonal dots. On an Alpha workstation as many as $24^{4}$ lattice points could be used, and in all cases we checked the convergence of the eigenvalues as the number of lattice points was increased to this level.

In Fig. 1 we present the ground-state charge distributions for the three types of polygonal boundary for a selection of dot sizes $L$, where $L$ is the side length of the polygon. In each case the dot material was taken to be GaAs, with an effective mass $m^{*}=0.067 m_{e}$, and a relative permittivity $\epsilon_{r}=10.9$, re-
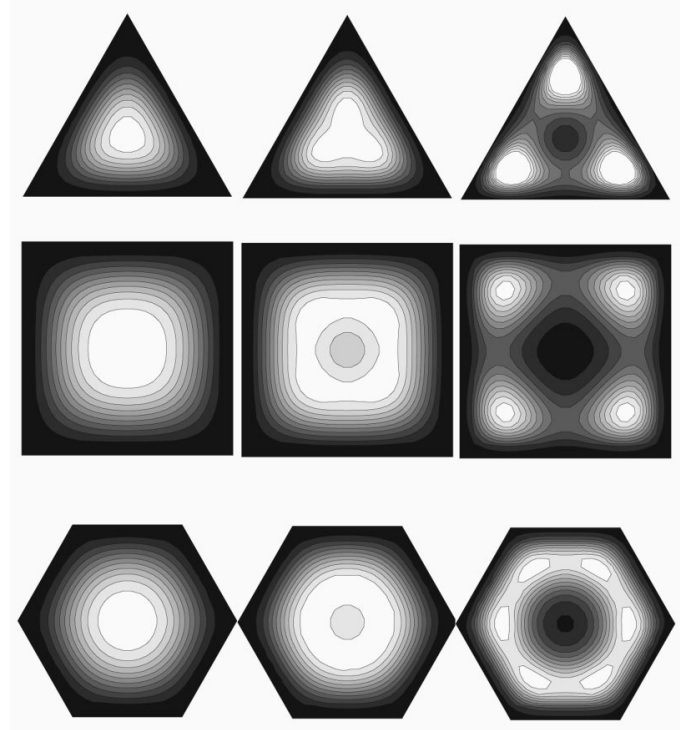

FIG. 1. Ground-state charge distributions for the three types of quantum dot. Dot sizes are (a) $50 \mathrm{~nm}$, (b) $100 \mathrm{~nm}$, and (c) $800 \mathrm{~nm}$.

sulting in a Bohr radius of $a_{\mathrm{B}}=8.8 \mathrm{~nm}$. For small dots the Coulomb interaction is weak on the scale of the kinetic energy, and the two-particle ground state resembles the noninteracting ground state, with the charge distribution being peaked at the center of the dot. Conversely, in very large dots the charge distribution is strongly localized near the vertices of the dot where the interaction is minimized, and the charge distribution practically vanishes away from these maxima. In analogy to the formation of a Wigner crystal in an infinite electron system, this configuration is referred to as a Wigner molecule. ${ }^{14}$ It is an important question to clarify the characteristic scale $r_{\mathrm{c}}$ of the mean-electron separation for which the crossover between these two extreme cases takes place, since the actual value of $r_{\mathrm{c}}$ cannot be obtained reliably using analytical arguments. In 1D boxes $r_{\mathrm{c}} \approx 1.5 a_{\mathrm{B}}$ has been found from tracing the charge-density distribution of the exact ground state. ${ }^{14}$ Using this technique we detect the change from the noninteracting situation by observing the point at which the charge distribution first shows a local minimum at the center of the dot instead of a maximum. In this way we estimate from Fig. 1 that $r_{\mathrm{c}} \approx 10 a_{\mathrm{B}}$ for all three dot geometries, which is almost an order of magnitude larger than the values found in one dimension. This rough estimate is in reasonable agreement with the critical value of $r_{\mathrm{c}} \approx 35 a_{\mathrm{B}}$ found by Ceperley for crystallization of the $2 \mathrm{D}$ electron gas. ${ }^{9}$ A somewhat larger value for $r_{\mathrm{c}}$ has been conjectured ${ }^{15}$ in two dimensions, as compared to one dimension, due to the considerably enhanced tendency for the electrons to surround one another. Although $r_{\mathrm{c}}$ marks the transition from the noninteracting regime, an additional qualitative change occurs at a somewhat longer length scale, at which the charge distribution develops $N_{0}$ sharply defined, well-separated maxima. It is to be expected that as the number of vertices is increased, and the polygon becomes closer to a circle, that this transition will occur at increasingly low densities. Accordingly, we can observe this change at $\sim 30 a_{\mathrm{B}}$ for the triangle and square, but only at the larger value of $\sim 100 a_{\mathrm{B}}$ for the hexagon. Before this transition occurs in the hexagonal dot 
the charge density shows a ringlike structure (as would occur in a circular dot), and in this regime the spectrum indeed resembles that of a diatomic rotor composed of spin $1 / 2$ fermions, as can be seen below in Fig. 6(b).

Figure 1 clearly shows how the electron localization takes place in sufficiently large dots around specific sites in real space. Anticipating this behavior motivated the mapping to a lattice model with a combination of hopping, exchange, superexchange, and Coulomb repulsion processes. In Ref. 11 a $t J V$ model:

$$
\begin{aligned}
H^{t J V}= & \mathcal{P}\left[\sum_{\langle i, j\rangle, \sigma}-t\left(c_{i \sigma}^{\dagger} c_{j \sigma}+\text { H.c. }\right)\right. \\
& \left.+J\left(\mathbf{S}_{i} \cdot \mathbf{S}_{j}-\frac{n_{i} n_{j}}{4}\right)+V n_{i} n_{j}\right] \mathcal{P}
\end{aligned}
$$

was proposed to describe the low-energy physics. Here $\mathcal{P}$ is a projector eliminating doubly occupied lattice sites, and $t$ and $J$ are the standard hopping and Heisenberg terms between nearest-neighbor sites. The $V$ term accounts for the nearest-neighbor Coulomb repulsion and may be written $V$ $\approx e^{2} / 4 \pi \epsilon_{0} \epsilon_{r} L$ for large $L$. Although first-principles calculations of the energies $t$ and $J$ are difficult, one can easily estimate the ordering of their magnitudes:

$$
|J| \ll|t| \ll V
$$

For the three geometries considered here $H^{t J V}$ can be diagonalized analytically to obtain the energy levels in terms of $t, J$, and $V$, together with their corresponding spins. Let us first consider the case of the square dot, which is described by two electrons moving on a four-site lattice. Here the $V$ term in Eq. (5) is important to discriminate energetically between two electrons sitting on diagonally opposite vertices as opposed to being on adjacent vertices. The lowest energy manifold of states was derived in Ref. 11 and, setting the ground-state energy to zero, the manifold consists of a singlet ground state, two degenerate triplet states at $(2 \Delta+J)$, and a singlet at an energy of $4 \Delta$ where $\Delta=2 t^{2} / V$, and $\Delta$ $\gg|J|$. The next set of levels are separated from this lowest energy multiplet by an energy gap of the order of $V$. The transition from the almost constant level separation found in small dots to a spectrum consisting of multiplets separated by relatively large energy gaps of this sort can be regarded as a signature for the crossover to the Wigner regime. ${ }^{16}$ Note, however, that the absolute level separations decrease rapidly with increasing dot size, as does the temperature scale for which this crossover might be observed.

In Fig. 2 we plot the lowest energy levels of the square dot as a function of the dot size, normalized to the energy of the highest singlet state. The overall structure of the spectrum agrees with that of the $t J V$ Hamiltonian (5), and quantitatively reproduces the numerically exact spectra obtained by Bryant for large dots. ${ }^{2}$ The doubly degenerate triplet state lies between the two singlet states and, asymptotically, the spectrum becomes equidistant as $L \rightarrow \infty$. Remarkably, the triplet levels are below $1 / 2$ for finite $L$ corresponding to a ferromagnetic $J<0$. This is somewhat unexpected, since pair exchanges of electrons would be expected to have antiferromagnetic couplings. ${ }^{17}$ However, the analysis presented in

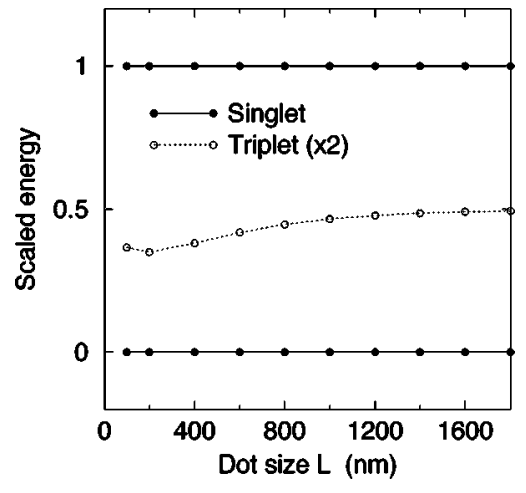

FIG. 2. Lowest energy levels for a square dot.

Ref. 11 does not a priori exclude negative values for the exchange coupling, since the direct exchange term might dominate the superexchange term. To exclude perturbative influences on the ground manifold arising from higher excited states that are ignored in the effective low-energy description, we plot the decay of $|J / \Delta|$ versus dot size in Fig. 3(a). The excellent straight line of the data in this semilogarithmic plot clearly suggests that $J \propto \Delta e^{-L / r_{\mathrm{c}}}$, as opposed to the power law that perturbative influences would give. The value of $r_{\mathrm{c}} \approx 52 a_{\mathrm{B}}$, as read off from Fig. 3(a), provides independent corroboration of the length scale characterizing the transition to the Wigner state estimated earlier from the charge-density distributions.

The dominant energy scale $\Delta$ can be roughly estimated using the pocket-state picture ${ }^{15}$ together with the WKB approximation:

$\Delta \sim \mathrm{e}^{-S_{0}}$,
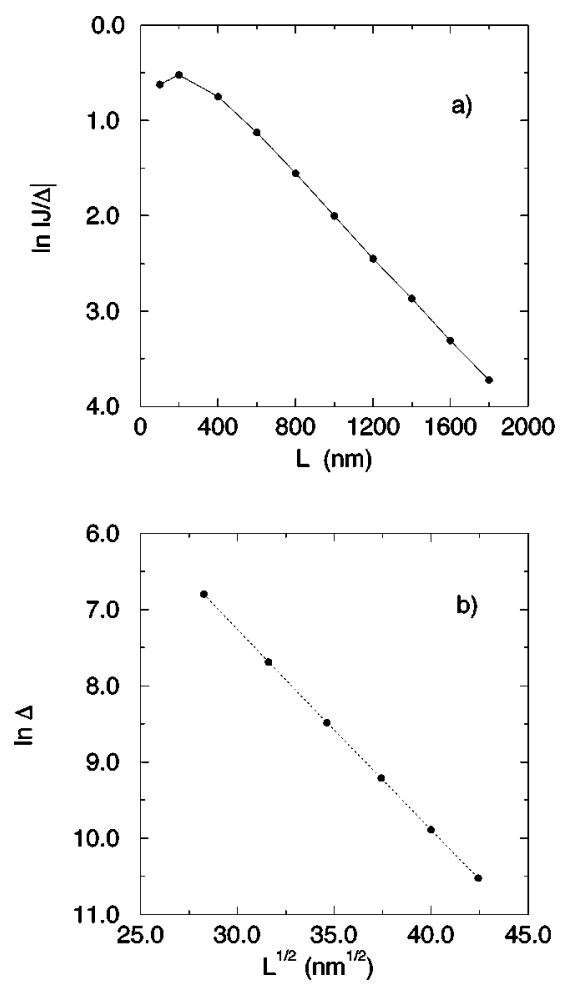

FIG. 3. (a) Decay of $J / \Delta$ with dot size $L$; (b) decay of $\Delta$ with $L$, line of best fit is shown. 


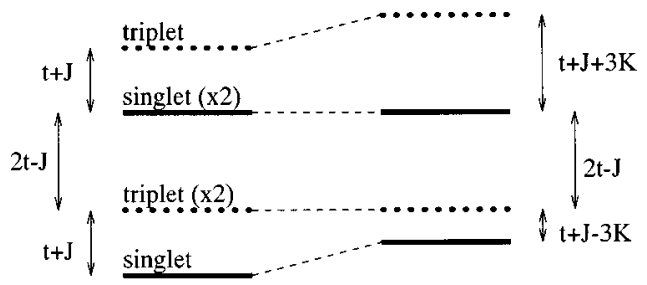

(a)

(b)

FIG. 4. (a) Energy-level structure obtained by solution of the $t J$ model. Level degeneracies are shown in brackets. (b) Modifications to the energy levels produced by the addition of the $K$ term (see text).

where the classical Euclidian action:

$$
S_{0}=\int_{1}^{2} \mathrm{~d} \vec{q} \sqrt{2 m n[v(\vec{q})-v(\overrightarrow{0})]}
$$

is taken along the path $\vec{q}:\left(\mathbf{r}_{1}^{(1)}, \mathbf{r}_{2}^{(1)}\right) \rightarrow\left(\mathbf{r}_{1}^{(2)}, \mathbf{r}_{2}^{(2)}\right)$ that carries $n$ electrons between two adjacent energy minimum configurations "(1)" and "(2)" ( $n=2$ for the example of the square and $n=1$ for the triangle). Here, $v(\vec{q})=e^{2} / 4 \pi \epsilon_{0} \boldsymbol{\epsilon}_{r}\left|\mathbf{r}_{1}-\mathbf{r}_{2}\right|$. For the square, the path corresponds to a $\pi / 2$ rotation of the two electrons around the center of the square, yielding:

$$
S_{0}^{\text {square }} \approx 0.79 \sqrt{r_{\mathrm{s}}} .
$$

The parameter $r_{\mathrm{s}}$ is the typical scale of electron separation (which for two electrons is equal to the dot size $L$ ). In Fig. 3 (b) we show $\Delta$ versus $\sqrt{L}$ on a semilogarithmic plot and verify that $\Delta \sim \mathrm{e}^{-\sqrt{L / \xi}}$. From the slope we extract a value for $\xi=1.64 a_{\mathrm{B}}$ that is in excellent agreement with the pocketstate prediction of $\xi=1.60 a_{\mathrm{B}}$ from Eq. (9).

The triangular dot is described by two electrons moving on a three-site lattice, and hence the $V$ term is irrelevant as it will just give rise to an overall shift in energy levels. The resulting $t J$ model has a more complicated ground-state manifold than for the square dot, and is shown in Fig. 4(a). This is also exactly the ground-state manifold for the hexagonal dot, where the $V$ term is again of importance. We can again employ the pocket state WKB theory to estimate the magnitude of the dominant energy scale $t$. For the triangle we have

$$
S_{0}^{\text {triangle }} \approx 0.42 \sqrt{r_{\mathrm{s}}}
$$

for one electron hopping along the edge to the empty site. In Fig. 5 we present a semilogarithmic plot of $t$ versus $\sqrt{L}$ for the triangle, which again confirms that the scaling of the dominant energy is of the form $t \sim \mathrm{e}^{-\sqrt{L / \xi}}$. The value of $\xi$ $=4.45 a_{\mathrm{B}}$ measured from this plot compares reasonably with $\xi=5.67 a_{\mathrm{B}}$ as predicted by the WKB theory from Eq. (10).

In Fig. 6 we present the energy levels obtained by the diagonalizations (again scaled by the energy of the highest singlet state) for the triangle and the hexagon. The sequence and the asymptotic ratios of the energy separations again agree with that predicted by the $t J V$ model. In contrast to the earlier result, however, it is not possible to fit these results with a single $J$. For example, for both geometries the higher triplet approaches its asymptotic value $4 / 3$ from above, implying $J>0$, whereas the lower triplet approaches $1 / 3$ from

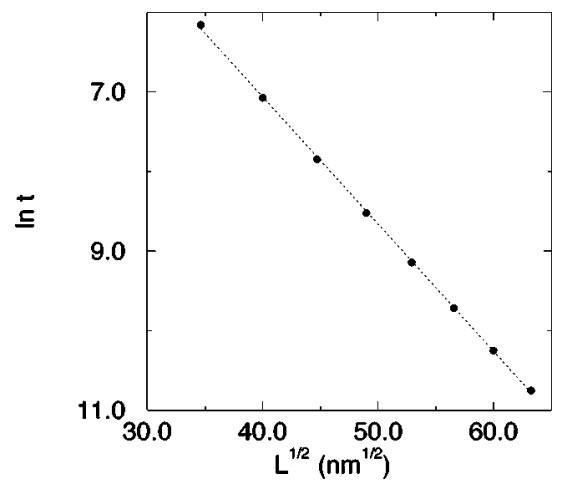

FIG. 5. Decay of $t$ with $L$ for a triangular dot, line of best fit is shown.

below, requiring a ferromagnetic $J$. This behavior indicates that although the $t J V$ Hamiltonian is able to adequately predict the gross features of the low-energy spectrum, it does not, in fact, provide a complete description of the dynamics occurring in the triangular and hexagonal dots. As this Hamiltonian was obtained as a reduced version of a more general model of Hubbard type ${ }^{11}$ this raises the possibility that during the reduction procedure some terms were dropped, which may be of importance in these situations. To investigate this further we again consider the lattice model with three sites forming an equilateral triangle and occupied by two electrons. With one orthogonal state per site the Hamiltonian is a generalized Hubbard model:
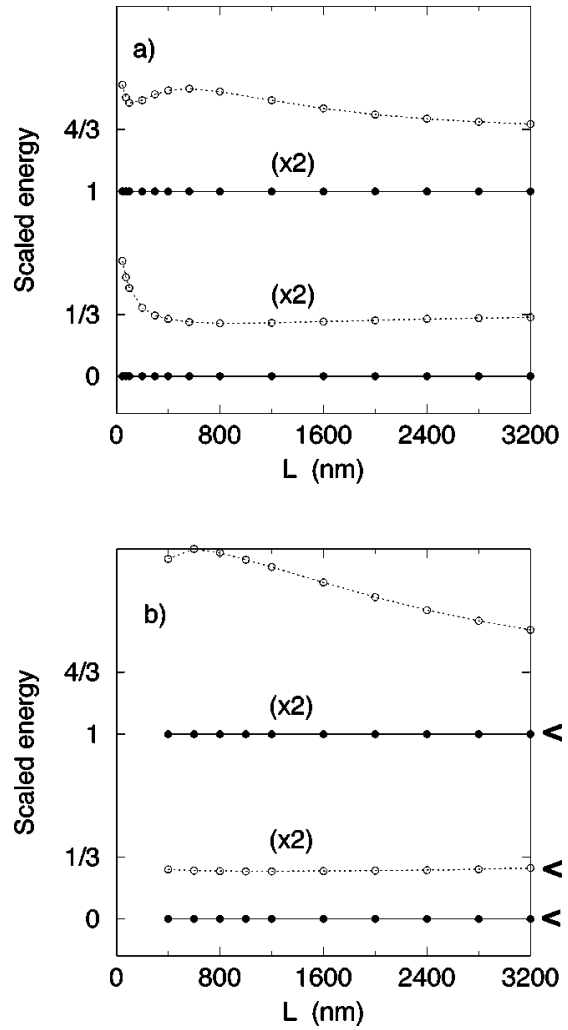

FIG. 6. Energy-level structures: denotes singlet states, $\bigcirc$ denotes triplet states. Level degeneracies are shown in brackets. (a) Triangular dot: the asymptotic decay to the values predicted by the $t J$ model is very evident. (b) Hexagonal dot: the decay to the asymptotic values is clearly less rapid than for the triangle. The arrows mark the energy levels of a rigid rotor system. 


$$
H=\sum_{i j \sigma} t_{i j} c_{i \sigma}^{\dagger} c_{j \sigma}+\frac{1}{2} \sum_{i j k l \sigma \sigma^{\prime}} U_{i j k l} c_{i \sigma^{\prime}}^{\dagger} c_{j \sigma^{\prime}}^{\dagger} c_{l \sigma^{\prime}} c_{k \sigma},
$$

where $t_{i j}$ are the one-electron matrix elements coming from the kinetic energy and the confining potential, and $U_{i j k l}$ are Coulomb matrix elements. We now consider all contributions to the low-lying manifold for which there is no double occupation of sites. (These are much higher in energy and their effect may be accounted for by second-order perturbation theory where they give rise to an antiferromagnetic superexchange term, as described in Ref. 11.) The largest intersite Coulomb term has matrix elements $U_{i j i j} \equiv V$ and the corresponding (ferromagnetic) exchange term has matrix elements $U_{i j j i} \equiv J_{\mathrm{F}} / 2$. These terms are already included in the $t J V$ Hamiltonian. All other terms in Eq. (11) involve hopping of electrons and fall into the following three classes:

(1) $(i=j=l: k \rightarrow j)$

$$
\frac{1}{2} \sum_{i j \sigma} U_{i i j i} c_{i \sigma}^{\dagger} c_{i \bar{\sigma}}^{\dagger} c_{j \bar{\sigma}} c_{i \sigma}=\frac{1}{2} \sum_{i j \sigma} U_{i i j i} n_{i \sigma} c_{i \bar{\sigma}}^{\dagger} c_{j \bar{\sigma}} .
$$

This is a spin-dependent hopping term which always takes us to a high-energy state since it involves double occupation (e.g., an electron hops from site 1 to site 2 provided site 2 is occupied with an electron of opposite spin). It thus contributes to superexchange by lowering the energy of singlets. This term, which renormalizes $J$, is also discussed in Ref. 11 .

(2) $(i=k: j \neq l \neq i)$

$$
\frac{1}{2} \sum_{i j l \sigma \sigma^{\prime}} U_{i j i l} c_{i \sigma}^{\dagger} c_{j \sigma^{\prime}}^{\dagger} c_{l \sigma^{\prime}} c_{i \sigma}=\frac{1}{2} \sum_{i k l \sigma} U_{i j i l} n_{i} c_{j \sigma}^{\dagger} c_{l \sigma} .
$$

This is potentially an important term since it operates in the ground manifold. However, for two electrons in a triangle, we can set $n_{i}=1$ (i.e., site $i$ is always occupied for states in which $c_{j \sigma}^{\dagger} c_{l \sigma}$ does not give zero) and hence this term merely renormalizes the kinetic energy $(t)$ term.

(3) $(i=l: j \neq k \neq i)$

$$
\begin{aligned}
\frac{1}{2} \sum_{i j k \sigma \sigma^{\prime}} U_{i j k i} c_{i \sigma}^{\dagger} c_{j \sigma^{\prime}}^{\dagger} c_{i \sigma^{\prime}} c_{k \sigma} & =-\frac{1}{2} \sum_{i j k \sigma \sigma^{\prime}} U_{i j k i} c_{i \sigma}^{\dagger} c_{i \sigma^{\prime}} c_{j \sigma^{\prime}}^{\dagger} c_{k \sigma} \\
& =-K \sum_{i j k \sigma \sigma^{\prime}} c_{i \sigma}^{\dagger} c_{i \sigma^{\prime}} c_{j \sigma^{\prime}}^{\dagger} c_{k \sigma}
\end{aligned}
$$

where the last step follows since all $U$ 's are the same ( $K$ $=U / 2$ ).

This last term is potentially important since it operates in the ground manifold and it cannot be accounted for simply as a renormalization of the other parameters (i.e., $J$ and $t$ ). It is also spin dependent, behaving differently when operating on singlet states from triplet states. Consider a base state in which there is a spin-up electron on site $i$, a spin-down electron on site $k$, and no electron on site $j$. This operator will move an electron from site $k$ to site $j$ and then flip the spins of sites $i$ and $j$. It is thus a combined hop and spin flip, as shown in Fig. 7(a). On the other hand, when it operates on a state where both electrons have the same spin it only performs the hop. Furthermore, an estimate of the magnitude of $K$ shows that it is comparable with $J$.

Retaining all terms gives the effective model:

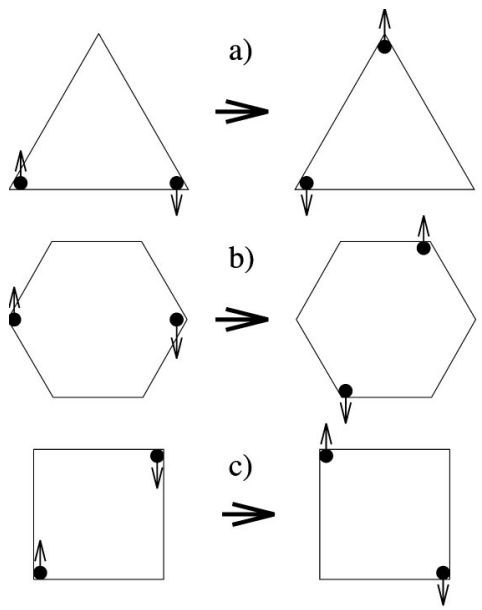

FIG. 7. K processes. (a) Triangular dot: hop followed by spin flip. (b) Equivalent process for hexagonal dot: $\pi / 3$ rotation followed by spin flip. (c) Square dot: $\pi / 2$ rotation followed by spin flip, equivalent to $-\pi / 2$ rotation without spin flip.

$$
\begin{aligned}
H^{\mathrm{eff}} & =H^{t J}-K \sum_{i j k \sigma \sigma^{\prime}} c_{i \sigma}^{\dagger} c_{i \sigma^{\prime}} c_{j \sigma^{\prime}}^{\dagger} c_{k \sigma} \\
& =H^{t J}-2 K \sum_{i j k \sigma}\left(\mathbf{S}_{i} \cdot \mathbf{S}_{j}+\frac{1}{4}\right) c_{j \sigma}^{\dagger} c_{k \sigma},
\end{aligned}
$$

where $K$ is a positive coupling and the $V$ dependence has been dropped since it gives the same energy contribution for all states. Introducing this additional term alters the eigenenergies as shown in Fig. 4(b), and this alteration is of exactly the correct form to account for the energy-level separations obtained from the numerical diagonalizations. For both dot geometries, fitting the results with these parameters yields a positive value for $K$ as expected, and a positive (antiferromagnetic) $J$ of similar magnitude.

We can also show that a $K$ term is also important for the low-lying manifold of other polygonal quantum dots. Consider, for example, the regular hexagonal geometry for which the exact numerical low-lying spectrum was described earlier. Starting with an extended Hubbard model and taking the "atomic" limit gives a 12-fold degenerate ground manifold of states, corresponding to three equivalent positions in which the two electrons are directly opposite each other and a factor of 4 for spin. In second order the degeneracy is partly lifted giving an effective Hamiltonian with "ring" terms, which correspond to a simultaneous rotation of the electrons by $\pm \pi / 3$ about the center of the dot. In fourth order we get the usual superexchange $(J)$ term but, in addition, we get a $K$ term that also corresponds to a simultaneous rotation of $\pm \pi / 3$ but now also involves a spin flip, as shown in Fig. 7(b). The final effective Hamiltonian has the form:

$$
\begin{aligned}
H^{\mathrm{eff}}= & \sum_{\langle i, j\rangle}\left[-\Delta\left(R_{\pi / 3}+R_{-\pi / 3}\right)+J\left(\mathbf{S}_{i} \cdot \mathbf{S}_{j}-\frac{1}{4}\right)\right. \\
& \left.-K\left(\mathbf{S}_{i} \cdot \mathbf{S}_{j}+\frac{1}{4}\right)\left(R_{\pi / 3}+R_{-\pi / 3}\right)\right] n_{i} n_{j},
\end{aligned}
$$

where the summation is over all three pairs of opposite sites, and the operator $R_{\theta}$ rotates the electrons by an angle $\theta$ about 
the center of the hexagon. The effective Hamiltonian (14) describing the low-lying states of the hexagon is isomorphic to the effective Hamiltonian for the triangular dot [Eq. (12)] with $\Delta$ playing the role of $t$. This may be seen explicitly by writing down expressions for the effective Hamiltonian matrices in their respective localized bases. Thus, the low-lying eigenstates of the triangular and hexagonal dots are in oneto-one correspondence, in agreement with the numerical results.

Note that by a similar reasoning we can generate effective $K$ terms for any polygonal dot. With the exception of the square dot, such terms are necessary in order to give quantitative agreement with the exact low-lying spectrum. This is not the case for the square since, as shown in Fig. 7(c), a rotation of $\pi / 2$ followed by a spin flip is equivalent to a rotation of $-\pi / 2$ with no spin flip. The effect is, therefore, to merely renormalize the second-order ring processes.

In conclusion, we have examined the behavior of the lowest energy levels of two electrons confined to twodimensional polygonal quantum dots. For sufficiently large dots the ground-state charge distribution shows a quasicrystalline structure, which can be used as the basis for mapping the system to an effective $t J V$ lattice model. This model is found to give the correct ordering of energy levels and to give a good first approximation to the energy-level spacings. The dominant energy scale of the systems can be estimated semiclassically to good accuracy, and although it is hard to obtain estimates for the remaining parameters, they decrease exponentially with the size of the dot and so this description becomes increasingly reliable in large dots. For the case of triangular and hexagonal dots, however, it was evident that it was necessary to retain the three-site terms previously neglected in the derivation of the effective model to account for the detailed behavior of the lowest energy levels. The lattice description of the dot considerably simplifies the calculation of the energy spectrum, and provides an appealing interpretation of the low-energy excitations occurring in these structures. It is still desirable to investigate different electron numbers and dot geometries to check the universality of our results concerning the critical density characterizing the crossover into the Wigner regime.

C.E.C. is grateful for financial support from the Leverhulme Foundation and W.H. acknowledges support by the Deutsche Forschungsgemeinschaft through SFB 276. EU support from the TMR program is also gratefully acknowledged.
${ }^{1}$ For reviews see, e.g., M. A. Kastner, Rev. Mod. Phys. 64, 849 (1992); L. P. Kouwenhoven, T. H. Oosterkamp, M. W. S. Danoeastro, M. Eto, D. G. Austing, T. Honda, and S. Tarucha, Science 278, 1788 (1997).

${ }^{2}$ G. W. Bryant, Phys. Rev. Lett. 59, 1140 (1987).

${ }^{3}$ U. Merkt, J. Huser, and M. Wagner, Phys. Rev. B 43, 7320 (1991).

${ }^{4}$ D. Pfannkuche, V. Gudmundsson, and P. A. Maksym, Phys. Rev. B 47, 2244 (1993).

${ }^{5}$ F. M. Peeters and V. A. Schweigert, Phys. Rev. B 53, 1468 (1996).

${ }^{6}$ J. Weis, R. J. Haug, K. von Klitzing, and K. Ploog, Phys. Rev. B 46, 12837 (1992).

${ }^{7}$ R. C. Ashoori, H. L. Stormer, J. S. Weiner, L. N. Pfeiffer, S. J. Pearton, K. W. Baldwin, and K. W. West, Physica B 184, 378
(1993); S. Tarucha, D. G. Austing, T. Honda, R. J. van der Hage, and L. P. Kouwenhoven, Phys. Rev. Lett. 77, 3613 (1996).

${ }^{8}$ E. P. Wigner, Phys. Rev. 46, 1002 (1934).

${ }^{9}$ B. Tanatar and D. M. Ceperley, Phys. Rev. B 39, 5005 (1989).

${ }^{10}$ S. T. Chui and B. Tanatar, Phys. Rev. Lett. 74, 458 (1995).

${ }^{11}$ J. H. Jefferson and W. Häusler, Phys. Rev. B 54, 4936 (1996).

${ }^{12}$ V. M. Bedanov and F. M. Peeters, Phys. Rev. B 49, 2667 (1994).

${ }^{13}$ L. F. Feiner, J. H. Jefferson, and R. Raimondi, Phys. Rev. B 51, 12797 (1995).

${ }^{14}$ K. Jauregui, W. Häusler, and B. Kramer, Europhys. Lett. 24, 581 (1993).

${ }^{15}$ W. Häusler, Z. Phys. B 99, 551 (1995).

${ }^{16}$ W. Häusler and B. Kramer, Phys. Rev. B 47, 16353 (1993).

${ }^{17}$ C. Herring, Rev. Mod. Phys. 34, 631 (1962). 